\title{
Women in Orthopaedic Fellowships: What Is Their Match Rate, and What Specialties Do They Choose?
}

\author{
Lisa K. Cannada MD
}

Published online: 13 April 2016

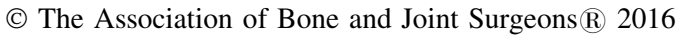

\begin{abstract}
Background Orthopaedic fellowship training is a common step before becoming a practicing orthopaedic surgeon. In the past, fellowship decisions in orthopaedics were made early in the residency and without a formal match. The process was disorganized, often not fair to the applicants or fellowship programs. More recently, there has been an organized match process for nine different disciplines in orthopaedics. Although the numbers of women applicants into orthopaedic residency has been reported and is the target of efforts to continue to improve gender diversity in orthopaedics, the numbers regarding women in orthopaedic fellowships have not been known. Other details including if there is a difference in match rate between male and female fellowship applicants and what discipline they choose to pursue across orthopaedic surgery has not been reported.

Questions/purposes (1) How have the numbers of women applying to orthopaedic fellowships changed over a 5-year period? (2) Is gender associated with fellowship match success? (3) Which subspecialties have greater proportions of female applicants?
\end{abstract}

The author has served as previous Chair of the OTA Fellowship \& Career Choices Committee (2007-2010) and Chair of the American Academy of Orthopaedic Surgeons Board of Specialty Societies Match Committee (2009-2015). The author is currently Board of Specialty Society Chair Elect and serves on the American Academy of Orthopaedic Surgeons Board of Directors (2015-2018).

All ICMJE Conflict of Interest Forms for authors and Clinical Orthopaedics and Related Research ${ }^{\mathbb{R}}$ editors and board members are on file with the publication and can be viewed on request.

L. K. Cannada ( $\square)$

Department of Orthopaedic Surgery, Saint Louis University,

3635 Vista Avenue, 7th Floor-Desloge Tower, St Louis,

MO 63110, USA

e-mail: LCannada@slu.edu
Methods Available orthopaedic residency match data regarding number of applicants and number of female residents between 2010 and 2014 were obtained. For fellowship data, our method was a review of the applicants who submitted rank lists and the number of applicants who matched in all subspecialties through San Francisco Match and from the American Shoulder and Elbow Society from 2010 to 2014. For each year, the number of females versus males applying was abstracted. The total number of females versus males who matched was then obtained. For each subspecialty represented in this article, the number of female applicants and matches was compared with the male applicants and matches.

Results The proportion of fellowship applicants who are female ranged from $7 \%$ to $10 \%$ annually, and the percentage of matched female applicants ranged from $8 \%$ to $12 \%$. Overall, combining results from 2010 to 2014, female fellowship applicants had a higher proportion of match success when compared with men (women: 320 of 335 [96\%]; men: 2696 of 3325 [81\%]; p < 0.001). Pediatric orthopaedic fellowships had the highest proportion of women (79 of 318 [25\%] followed by foot and ankle (42 of 311 [14\%]; spine had the lowest (15 of 525 [3\%]).

Conclusions Women applicants for advanced orthopaedic training matched at a higher proportion than men in fellowship training. Pediatrics has a higher proportion of women applicants and fellows. Orthopaedics should be a model for other surgical specialties by encouraging women to successfully pursue advanced training.

\section{Introduction}

Orthopaedics is a specialty that has been largely comprised of males $[10,16]$. Strides have been made in recruiting 
female residents. In the past 5 years, female residents account for anywhere from $12 \%$ to $15 \%$ of orthopaedic residents [2-6]. When accounting for the increasing number of residency positions, the actual number of female residents is steadily increasing [2-6] (Table 1). Completion of a fellowship is more often the norm for most orthopaedic residents. The exact number of residents pursuing an advanced training is estimated to be over $90 \%$ [7, 8, 14]. Because fellowship training is now through an organized match process, there has been accurate data on fellowship trends across the subspecialties since 2010 .

What has not been reported is the breakdown of women fellowship applicants, their match rate, and specialty choices. With more residents than ever doing fellowships, and often times more applicants than positions [7], does the role of gender in fellowship training matter? This is an important topic to address as residents are mentored regarding career choices and the future workforce distribution in orthopaedics may be affected.

We therefore asked: (1) How have the numbers of women applying to orthopaedic fellowships changed over a 5-year period? (2) Is gender associated with fellowship match success? (3) Which subspecialties have greater proportions of female applicants?

\section{Materials and Methods}

Gender data on female orthopaedic residents were obtained from the Journal of the American Medical Association for 2010 to 2014. The numbers reporting the female matched applicants in orthopaedic surgery is available through the National Resident Matching Program (NRMP) data. The percentage of orthopaedic positions filled by women were those who designated themselves as "female" on the NRMP application. Although the phenomena we are studying here clearly deal with gender issues-that is, the constellation of sociocultural constructs that interact with biological sex-rather than sex itself (a purely biological phenomenon referring to a particular genotype) [12], and so the terms used to discuss these phenomena would normally be man/woman rather than male/female, we are

Table 1. Proportion of orthopaedic match positions filled by females

\begin{tabular}{ll}
\hline Year & Females matched/total applicants \\
\hline 2010 & $78 / 653(12 \%)$ \\
2011 & $80 / 668(12 \%)$ \\
2012 & $89 / 682(13 \%)$ \\
2013 & $90 / 691(13 \%)$ \\
2014 & $97 / 693(14 \%)$ \\
\hline
\end{tabular}

choosing to use male/female where we report our results because that is how the source data are reported from the residency match program. Elsewhere in the article, we use the terms men and women, which is more correct for a discussion of gender-based phenomena.

Orthopaedic fellowship training has three organizations for nine different matches. Adult reconstruction/tumor, foot and ankle, pediatrics, spine, sports medicine, and trauma all use San Francisco (SF) Match for their fellowship match process. The American Shoulder and Elbow Society (ASES) currently administers its own match. Hand fellowships are obtained through the NRMP and include applicants from general surgery, plastic surgery, and orthopaedic surgery residencies. Contact was made with SF Match (San Francisco Match Program, Dennis S. Thomatos, Manager, data provided July 30, 2015, and October 28, 2015) and the ASES (Stella Gauthier, Society Coordinator, November 2, 2015) to obtain the match data for 2010 to 2014. The data for the match have been collected on an annual basis since 2010 through the American Academy of Orthopaedic Surgeons (AAOS) Board of Specialty Societies Match Committee. There are multiple data points available, including number of programs, positions, number of applicants submitting rank lists, and number of applicants matched. In addition, substantial additional data have been reported regarding the number of programs applied for by the applicants and the number of programs they rank: the number of applicants that match into their first choice and second choices, the number of applicants a program ranks, and the number of programs that did not match. Match result data by gender for similar data regarding applicants for orthopaedic surgery positions by orthopaedic residents specifically were not available for hand surgery.

The applications for SF Match do not list gender on their application. The applicant numbers were provided by SF Match based on first names. When female names were unclear, the letters of recommendation were used because the gender was clear in the letters. For the ASES match, again gender is not listed on the application. In cases in which there were questions, gender was confirmed through their database. The number of applicants by gender was then reported and data collated by female applicants, females who matched, male applicants, males who matched, and then further subdivided into the seven categories of specialties. The tumor match represents a small number of programs (16) and has been run with the adult reconstruction (hip and knee) match since the organized match process with SF Match began in 2010. The match results were not separated into tumor and adult reconstruction until 2015; thus, tumor as a specific subspecialty could not be reported for this article. 


\section{Statistical Analysis}

The data were compiled and then analyzed by each year and specialty to determine the number of female applicants and their match rate. Chi-square analysis was used to compare the values between gender for all data. Significance was set at $\mathrm{p}<0.05$.

\section{Results}

For fellowship applications, the proportion of female applicants ranged from $7 \%$ to $10 \%$ annually, and percentage of matched female applicants ranged from $8 \%$ to $12 \%$ (Table 2). In 2010, 9\% (60 of 674) of applicants were women, and of those who matched, $10 \%$ (56 of 582) were women. In 2011, $9 \%$ (65 of 688) of applicants were women, and of those who matched, $11 \%$ (62 of 571) were women. In $2012,7 \%$ (54 of 755) of applicants were women, and of those who matched, $8 \%$ ( 49 of 602 ) were women. In 2013, $10 \%$ (76 of 757) of applicants were women, and of those who matched, $12 \%$ (74 of 614) were women. In 2014, 10\% (80 of 786) of applicants were women, and of those who matched, $12 \%$ (79 of 647) were women.

Combining the 2010 to 2014 fellowship match years' data, female fellowship applicants had a higher proportion of match success when compared with men (women: 320 of 335 [96\%]; men: 2696 of 3325 [81\%]; $p<0.001$ ). The match rate for female applicants from 2010 to 2014 ranged from $91 \%$ to $99 \%$. The match rate for male applicants during that same time period ranged from $79 \%$ to $86 \%$
(Table 3). In 2010, four of 60 female applicants did not match. In 2011, three of 65 female applicants did not match. In 2012, five of 54 female applicants did not match. In 2013, two of 76 of female applicants did not match and in 2014, only one of 80 female applicants did not match. In 2010, 88 male applicants out of 614 did not match. In 2011, 114 male applicants out of 623 did not match. In 2012, 148 male applicants out of 701 did not match. In 2013, 141 male applicants out of 681 did not match. In 2014, 138 male applicants out of 706 did not match.

Pediatrics had the highest proportion of female applicants (79 of 318 [25\%]) followed by foot and ankle (42 of 311 [14\%]), shoulder (22 of 219 [10\%]), sports (94 of 1035 [9\%]), trauma (38 of $441[9 \%]$ ), hip and knee/tumor (45 of 789 [6\%]), and spine (15 of 525 [3\%]) (Table 4). In terms of absolute numbers, the most popular specialty is sports (94 females) followed by pediatrics (79), hip and knee/tumor (45), foot and ankle (42), trauma (38), shoulder (22), and spine (15).

\section{Discussion}

The number of women in medical school through residency and especially in surgery training has been evaluated over the years. We do know that women comprise over half the medical school class sizes, but women in orthopaedic surgery comprise no more than $15 \%$ of residents $[11,16]$. Thus, orthopaedics has often been criticized as having low percentages of women residents [16]. Regarding the AAOS membership, there are 1273 women allopathic members,

Table 2. Female fellowship applicant numbers

\begin{tabular}{|c|c|c|c|c|c|}
\hline Year & 2010 & 2011 & 2012 & 2013 & 2014 \\
\hline \multirow[t]{2}{*}{ Female applicants/total applicants } & $60 / 674$ & $65 / 688$ & $54 / 755$ & $76 / 757$ & $80 / 786$ \\
\hline & $9 \%$ & $9 \%$ & $7 \%$ & $10 \%$ & $10 \%$ \\
\hline \multirow[t]{2}{*}{ Females matches/total matched } & $56 / 582$ & $62 / 571$ & $49 / 602$ & $74 / 614$ & $79 / 647$ \\
\hline & $10 \%$ & $11 \%$ & $8 \%$ & $12 \%$ & $12 \%$ \\
\hline
\end{tabular}

Table 3. Match rate by gender

\begin{tabular}{llll}
\hline Year & Women & Men & p value \\
\hline 2010 & $56 / 60(93 \%)$ & $526 / 614(86 \%)$ & 0.090 \\
2011 & $62 / 65(95 \%)$ & $509 / 623(82 \%)$ & $<0.001$ \\
2012 & $49 / 54(91 \%)$ & $553 / 701(79 \%)$ & 0.040 \\
2013 & $74 / 76(97 \%)$ & $540 / 681(79 \%)$ & $<0.001$ \\
2014 & $79 / 80(99 \%)$ & $568 / 706(86 \%)$ & $<0.001$ \\
Overall (all years) & $320 / 335(96 \%)$ & $2696 / 3325(81 \%)$ & $<0.001$ \\
\hline
\end{tabular}


Table 4. Subspecialty applicant numbers for females

\begin{tabular}{llcl}
\hline Subspecialty & Female applicants & Total applicants & Percentage \\
\hline Pediatrics & 79 & 318 & 25 \\
Foot & 42 & 311 & 14 \\
Shoulder & 22 & 241 & 10 \\
Sports & 94 & 1035 & 9 \\
Trauma & 38 & 441 & 9 \\
Hip/knee/tumor & 45 & 789 & 6 \\
Spine & 15 & 525 & 3 \\
\hline
\end{tabular}

representing 5\% of members (Lucy Free, Representative, AAOS Member Services, July 20, 2015). What has not been reported or known was information on women in orthopaedic fellowship training. Therefore, we aimed to evaluate applications and match data, specifically looking at the proportion of women applying and matching to fellowships.

Our analysis was limited by the fact that fellowship applications do not ask for the applicant to report his or her gender. This study was based on names that appear to be women's names. For all data from SF Match, for the names that were unclear, the gender was confirmed by reviewing the letters of recommendation because gender was clear in the letters. For data from the ASES, in cases in which the names may have been unclear, gender was confirmed in the database. We believe the extensive amount of effort to determine gender leaves little room for error. We have accurate data from eight of the nine specialties in the orthopaedic fellowship match. There already is a paper referenced in this article with results of females in hand fellowships [1]. The details of data we used for our article were not available to us through the NRMP results. We feel that our results are a representation of female applicants in the fellowship process with eight of nine specialties and the first article to report on these results from all other specialty matches.

Another limitation is we cannot compare the number of residents applying for fellowships based on assumed year they entered residency. One must keep in mind the number of residents entering in 2010 would be applying for fellowship in 2013 if all are in a 5-year residency. When reviewing data from SF Match, in any given year of fellowship applicants, there are multiple years listed that the applicants began residency. This could be the result of military commitments, choosing a second fellowship, or a change in their career path. In any given year, there are people applying from more than 1 specific year of residency matching.

Women still comprises a minority of applicants to fellowship and of those who ultimately match. Our findings suggest that although there already are few women in orthopaedic residency (12-14\%, Table 1), fewer still apply to fellowships. Although it is reported more than $90 \%$ of orthopaedic residents do fellowships [7, 8, 13, 14], there are no accurate sources to confirm actual numbers of residents pursuing fellowships. Over the past several years, the number of residents applying for orthopaedic fellowships has been increasing in number [7]. Although it is difficult to ascertain exact reasons for pursuing specific fellowships, the work week limitations, deficiencies in one's training program, and desire for a specific specialty training to make one a desirable job applicant could contribute to the rationale for completing fellowships. An article that discussed trends in the orthopaedic job market and the importance of fellowship subspecialty training indicated the percentage of job opportunities advertised in an orthopaedic journal increased from $16.7 \%$ in 1984 to $68.2 \%$ in 2009 [13]. This indicates fellowship training can be helpful in making one a marketable job candidate. When considering the numbers are lower for women applying to fellowships, no statistical conclusions can be drawn as a result of limitations discussed here. However, the raw numbers increased from 54 in 2012 to more robust numbers of 76 women applicants in 2013 and 80 in 2014. This parallels the increase in women residents. Only by increasing the number of women residents will the percentages of women fellowship applicants increase.

We found females have a higher chance of a successful match to fellowship when compared with male applicants. In a paper on whether men outperform women during orthopaedic residency training, it was found for 90 residents in a program, there was no difference between male and female performance, and therefore performance, during residency should not bias any future selection for residency [15]. This also seems to be applicable for applying for fellowships. The performance of orthopaedic fellows has not been reported. We do not know the exact criteria in terms of gender differences in selecting a fellow. There are limited data on orthopaedic fellowship selection criteria by program directors. We do know a survey of 475 surgery fellowship program directors was competed and they found the most important criteria for selecting an applicant for an interview were a letter of recommendation from the subspecialty faculty, quality of residency program, and letters of recommendation from residency program directors [8]. However, when it came time for the rank list, the most important criteria were the interview, the letter of recommendation from the subspecialty faculty, from the residency program director, and then expressed interest in the program [8]. Interestingly, there are rules by each specialty set that limit postinterview communication, which could be an expression of interest in a 
program desired by the program directors. Our study found a higher match rate of female applicants to fellowship. It would be interesting to know the role gender plays in compiling rank lists.

Pediatrics as a subspecialty is well represented by women. This is supported in the article on women in orthopaedic academic practices. The highest percentage of respondents was in pediatric orthopaedic surgery, representing $42 \%$ of surgeons [9]. Having a high number of women role models in academic practice may be a reason for a high percentage of women applicants in pediatric orthopaedic fellowships. In terms of raw numbers, sports has the highest number of women applicants and matched individuals, and this is reflected by the large number of positions available. There is variation in numbers and percentages of women applicants into orthopaedic subspecialty training. Spine has the lowest numbers of any subspecialty. The match process is handled by the subspecialty societies. Perhaps early subspecialty educations and/or programs aimed at residents in conjunction with a mentoring program can be a resource not only to spur specific specialty fellowship interest, but also promote increased applications from women.

The opportunities for women matching in a fellowship are very high. There is variability in women's representation across all fellowship subspecialties. This information may be useful in counseling women in career choices and developing strategies to encourage these applicants to consider those specialties with less representation of women. Orthopaedics should be a model for other surgical specialties in terms of encouraging women to subspecialize in the field, because the match rates are higher for women.

Acknowledgments We thank Stella Gauthier, Society Coordinator for American Shoulder and Elbow Surgeons, and Dennis Thomatos, Manager, San Francisco Match Program, for providing the data on female applicants for fellowship matches. We also thank Heidi Israel $\mathrm{PhD}$, Saint Louis University Department of Orthopaedic Surgery, for her assistance with statistical analysis.

\section{References}

1. Bae GH, Lee AW, Park DJ, Maniwa K, Zurakowski D; ASSH Diversity Committee, Day CS. Ethnic and gender diversity in hand surgery trainees. J Hand Surg Am. 2015;40:790-797.

2. Brotherton SE, Etzel SI. Graduate medical education, 2009-2010. JAMA. 2010;304:1255-1270.

3. Brotherton SE, Etzel SI. Graduate medical education, 2010-2011. JAMA. 2011;306:1015-1030.

4. Brotherton SE, Etzel SI. Graduate medical education, 2011-2012. JAMA. 2012;308:2264-2279.

5. Brotherton SE, Etzel SI. Graduate medical education, 2012-2013. JAMA. 2013;310:2328-2346.

6. Brotherton SE, Etzel SI. Graduate medical education, 2013-2014. JAMA. 2014;312:2427-2445.

7. Cannada LK, Luhmann SJ, Hu SS, Quinn RH. The fellowship match process: the history and a report of the current experience. J Bone Joint Surg Am. 2015;97:e3(1-7).

8. Grabowski G, Walker JW. Orthopaedic fellowship selection criteria: a survey of fellowship directors. J Bone Joint Surg Am. 2013; 95:e154(1-6).

9. Hill JF, Johnson AH, Cannada L. A profile of female academic orthopaedic surgeons. Curr Orthop Pract. 2013: 24:636-640.

10. Hill JF, Yule A, Zurakowski D, Day CS. Residents' perceptions of sex diversity in orthopaedic surgery. J Bone Joint Surg Am. 2013;95: e144(1-6).

11. Huntington WP, Haines N, Patt JC. What factors influence applicants' rankings of orthopaedic surgery residency programs in the National Resident Matching Program? Clin Orthop Relat Res. 2014;472:2859-2866.

12. Leopold SS, Beadling L, Dobbs MB, Gebhardt MC, Lotke PA, Manner PA, Rimnac CM, Wongworawat MD. Fairness to all: gender and sex in scientific reporting. Clin Orthop Relat Res. 2014;472:391392.

13. Morrell NT, Mercer DM, Moneim MS. Trends in the orthopedic job market and the importance of fellowship subspecialty training. Orthopedics. 2012;35e 555-560.

14. Niesen MC, Wong J, Ebramzadeh E, Sangiorgio S, SooHoo NF, Luck JV, Eckardt J. Orthopedic surgery fellowships: the effects of interviewing and how residents establish a rank list. Orthopedics. 2015;38:175-179.

15. Pico K, Gioe TJ, Vanheest A, Tatman PJ. Do men outperform women during orthopaedic residency training? Clin Orthop Relat Res. 2010;468:1804-1808.

16. Van Heest AE, Agel J. The uneven distribution of women in porthopaedic residency training programs in the United States. J Bone Joint Surg Am. 2012;18:e9(1-8). 